\title{
Función Renal en Recién Nacidos de Término y Pretérmino Durante el Segundo Día de Vida(*)
}

\author{
Dr. Fernando Olavarría U. ${ }^{1}$; Dr. Sergio Krause H. ${ }^{1}$; Dr. Luis Barranco C. ${ }^{1}$; \\ E. U. Carmen Harding I. 2; T.M. María I. López N. ${ }^{3}$
}

Renal Function in Preterm and Term Infants

\begin{abstract}
Glomerular filtration rate (GFR) by means of creatinine clearence was measured in 42 newborn infants, whose gestational ages (G.A) ranged from 26 to 41 weeks. Renal excretion of water, $\mathrm{Na}^{+}$and $\mathrm{K}^{+}$were also measured. A direct correlation was find between gestational age (GA) and creatinine clearence ( $r$ : 0.67 ). An inverse correlation ocurred between GA and $\mathrm{Na}^{+}$fraccional excretion $(\mathrm{r}:-0.84)$, GA and plasma $\mathrm{K}^{+}$concentration (r: -0.67 ), but no correlation was find among GA and $\mathbf{K}^{+}$urinary excretion. Urine's volume and water excretion were also inversely related to GA (s; 0.83 and $r$ : -0.54 respectively).

(Key words: Creatinine Clearence. Glomerular filtration rate. Renal function. Term and preterm newborn infants. Water, Sodium, Potasium, excretion).
\end{abstract}

El progresivo interés por el estudio de la función renal en el recién nacido de diferente edad gestacional y los cambios que sobre ella impone la vida extrauterina ha levado, en los últimos años, a un conocimiento más preciso de la fisiologia del rix́ón en desarrollo.

Las investigaciones realizadas, se han orientado fundamentalmente a evaluar la velocidad de filtración glomerular y sus modificaciones en la vida postnatal ${ }^{1-12}$, asi como al estudjo de algunas funciones del transporte de agua y electrolitos $3,4,8,9,13-22$.

Lo avanzado hasta ahora, ha permitido un mejor enfoque terapéutico del recién nacido crítico, especialmente del pretérmino, contribuyendo a mejorar sustancialmente las tasas de morbimortalidad de este grupo.

En el presente estudio, se analizan algunos de los parámetros de función renal en recién nacidos normales de término y pretérmino antes de los dos días de vida, dada la falta de referencias nacionales sobre esta materia.

1. Instîtuto de Pediatría, Facultad de Medicina, Universidad Austral de Chile.

2. Servicio de Pediatría, Unidad de Neonatología, Hospital Regional, Valdivia.

3. Laboratorio de Nefrología, Facultad de Medicina, Universidad Austral de Chile, '

* Trabajo realizado con apoyo de la Dürección de Investigación y Desarrollo, Liniversidad Austral de Chile, Proyecto $\$-83-11$.

\section{MATERLAL Y METODO}

Se incluyeron 42 recién nacidos normales de ambos sexos, de diferente edad gestacional, entre las 24 y 48 horas de vida. La edad gestacional fue asignada de acuerdo a historia menstrual y evaJuación pediátrica según pauta de Dubowitz y Dubowitz ${ }^{23}$. Para los efectos de esta investigación se seleccionaron recién nacidos sanos, sin antecedentes de hipoxia perinatal, malformaciones, $u$ otras afecciones que pudieran interferir con el estudio.

La alimentación y la administración de soluciones electrolíticas fueron establecidas para cada caso en particular, aportandose durante el período de estudio, un volumen mínimo de 80 $\mathrm{ml} / \mathrm{kg}$ idía de liquidos como leche maternizada (NAN间) o solución glucosalina 1/4 nomal por vía endovenosa o ambas.

Al cumplir 24 horas de vida y previa compresión vesical, se instaló un recolector urinario pediátrico autoadhesivo (Terumoß) para colección y medición de diuresis en 24 horas. En el tiempo medio del período de colección de orina, se tomó una muestra de sangre venosa periférica para análisis de electrolitos y creatinina. El estudio de electrolitos plasmáticos y urinarios se realizó mediante un fotómetro de llama PerkinElmer. La determinación de creatinina en sangre y orina fue practicada mediante análisis fotométrico según técnica de Jaffe. La osmolaridad plasmática y urinaria fue determinada según 
punto de congeiación, usando un osmómetro Knauer.

La depuración de creatinina, excreción fraccional de sodio y depuración de agua libre fueron calculados según fórmulas convencionales.

El análisis estadístico de los datos se realizó con un computador Digital 2020, utilizando el paquete estadistico SPSS. La medida de tendencia central se estableció mediante promedio y desviación estándar ( $\mathrm{X} \pm \mathrm{D} . \mathrm{E}$.). Se utilizó la prueba $T$ de Student para muestras independientes y el análisis de correlación de Pearson con su curva de regresión correspondiente, fijándose la probabilidad de error en menos de $5 \%^{24}$.

Se solicitó la autorización materna para la incorporación de cada recién nacido a este estudio.

\section{RESULTADOS}

De los 42 recién nacidos que comprende este estudio, 26 corresponden al sexo masculino. La edad gestacional ( $\overline{\mathrm{X}} \pm \mathrm{D}$.E.) fue de $36 \pm 4.1$ semanas (rango: 26-41), y el peso de 2.461 \pm $1.017 \mathrm{~g}$. (rango: $820-4.920$ ); 20 eran zecién nacidos de término (RNT) ( 38 a 42 semanas de gestación) y 22 eran recién nacidos de pretérmino (RNPT). Para este último grupo la edad gestacional fue de $32.8 \pm 3.0$.

Del total de los recién nacidos estudiados, 35 tueron adecuados, 3 pequeños y 4 grandes para su edad gestacional, según estándar de Battaglia y Lubchenco 25 .
En la Tabla 1 se resumen los resultados del estudio para los grupos RNT y RNPT.

La depuración de creatinina fue significativamente más alta en el grupo RNT. Al corregir por superficie corporal, el resultado promedio de este grupo fue aproximadamente $1 / 4$ del valor correspondiente al estándar del adulto.

La relación entre depuración de creatinina y edad gestacional (Figura 1) fue directa $y$ significativa ( $r: 0.671 ; p<0.00001$ ). Sin embargo, al analizarla por tramo de edad gestacional esta se pierde después de las 34 semanas (r: 0.326 ; p: n.s.). La correlación entre peso de nacimiento y depuración de creatinina también fue directa y significativa (r: $0.855 ; p<0.00001$ ), pero se mantuvo incluso después de las 34 semanas (r: $0.760 ; \mathrm{p}<0.0001$ ).

La excreción fraccional de sodio urinario $\left(\mathrm{FE}_{\mathrm{Na}}\right.$ ) en el grupo RNT fue 10 veces menor que en el grupo RNPT, aunque no hubo diferencía en los valores de sodio plasmático en ambos grupos. La correlacción entre $\mathrm{FE}_{\mathrm{Na}}$ y edad ges: tacional fue inversa y significativa (r: -0.838 ; $\mathrm{p}<0.00001$ ). (Figura 2).

El nivel de potasio plasmático fuse significativamente más alto en el grupo RNPT, existiendo una relación inversa con edad gestacional ( $r:-0.667 ; p<0.0001$ ) (Figura 3). Sin embargo, no existieron diferencias en excreción urinaria de potasio (UKV) entre ambos grupos ni se pudo establecer una relación significativa entre edad gestacional y esta última variable.

El volumen urinario fue significativamente

TABLA I

RESUMEN ESTUDIO FUNCIONAL RENAL EN RNT Y RNPT ENTRE LAS 24 Y 48 HORAS DE VIDA.

\begin{tabular}{|c|c|c|c|}
\hline & $\begin{array}{l}\text { PRETERMINO } \\
(n=22)\end{array}$ & $\begin{array}{l}\text { TERMINO } \\
(n=20)\end{array}$ & $\mathrm{P}$ \\
\hline Ingresos $(\mathrm{ml} / \mathrm{kg} / \mathrm{día}$ & $92.74 \pm 4.34$ & $95.68 \pm 3.24$ & N.S. \\
\hline Depuración creatinina ( $\mathrm{ml} / \mathrm{min})$ & $1.74 \pm 0.15$ & $3.47 \pm 0.31$ & $<0,0001$ \\
\hline Depuración creatinina $\left(\mathrm{ml} / \mathrm{min} / 173 \mathrm{~m}^{2}\right)$ & $23.76 \pm 1.54$ & $29.32 \pm 2.13$ & $<0.05$ \\
\hline Sodio plasmático (mEq/lt) & $137.10 \pm 1.60$ & $135.40 \pm 1.60$ & N.S. \\
\hline Excreciön fraccional de sodio (\%) & $1.19 \pm 0.23$ & $0.14 \pm \quad 0.03$ & $<0.000 .1$ \\
\hline Potasio plasmático $(\mathrm{mEq} / \mathrm{lt})$ & $4.61 \pm 0.18$ & $3.92 \pm 0.14$ & $<0.005$ \\
\hline Excreción urinaria de potasio ( $\mathrm{mEq} / 24$ hrs.) & $1.62 \pm 0.14$ & $1.36 \pm 0.15$ & N.S. \\
\hline Osmolaridad plasmática $\left(\mathrm{mOsm} / \mathrm{kg} \cdot \mathrm{H}_{2} \mathrm{O}\right)$ & $262.80 \pm 2.70$ & $266.70 \pm 2.80$ & N.S. \\
\hline Volumen urinario (ml/kg/hora) & $3.55 \pm 0.26$ & $1.19 \pm 0.10$ & $<0.0001$ \\
\hline Osmolaridad urinaria $\left(\mathrm{mOsm} / \mathrm{kg} \cdot \mathrm{H}_{2} \mathrm{O}\right)$ & $135.40 \pm 8.40$ & $181.10 \pm 13.40$ & $<0.005$ \\
\hline Depuración agua libre (x $100 \mathrm{ml}$ VFG) & $0.57 \pm 0.22$ & $0.23 \pm 0.18$ & $<0.0001$ \\
\hline
\end{tabular}




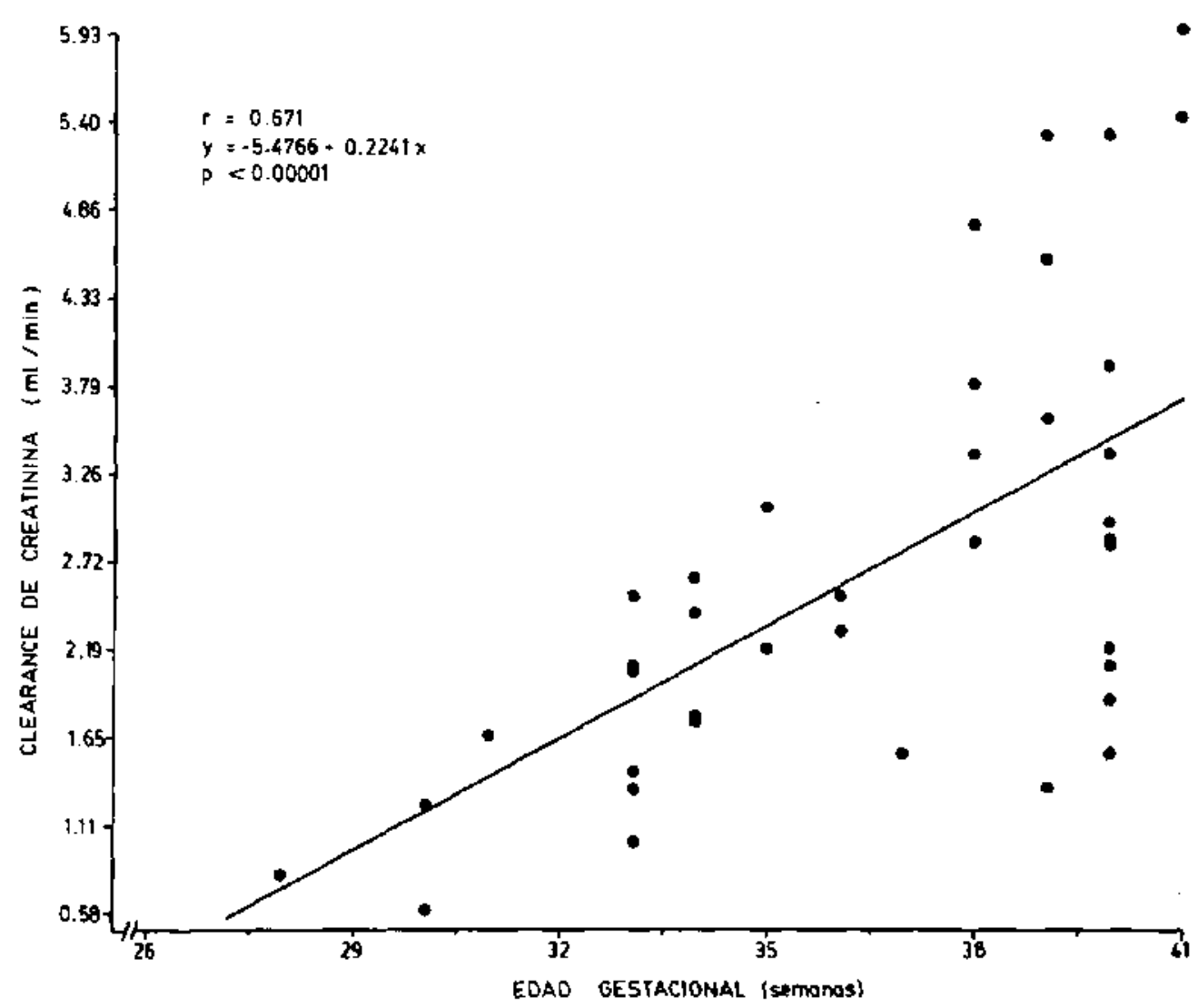

Figura 1: Correlación de Edad Gestacional y Depuración (clearence) de Creatinina antre las $24-48$ horas de vida.

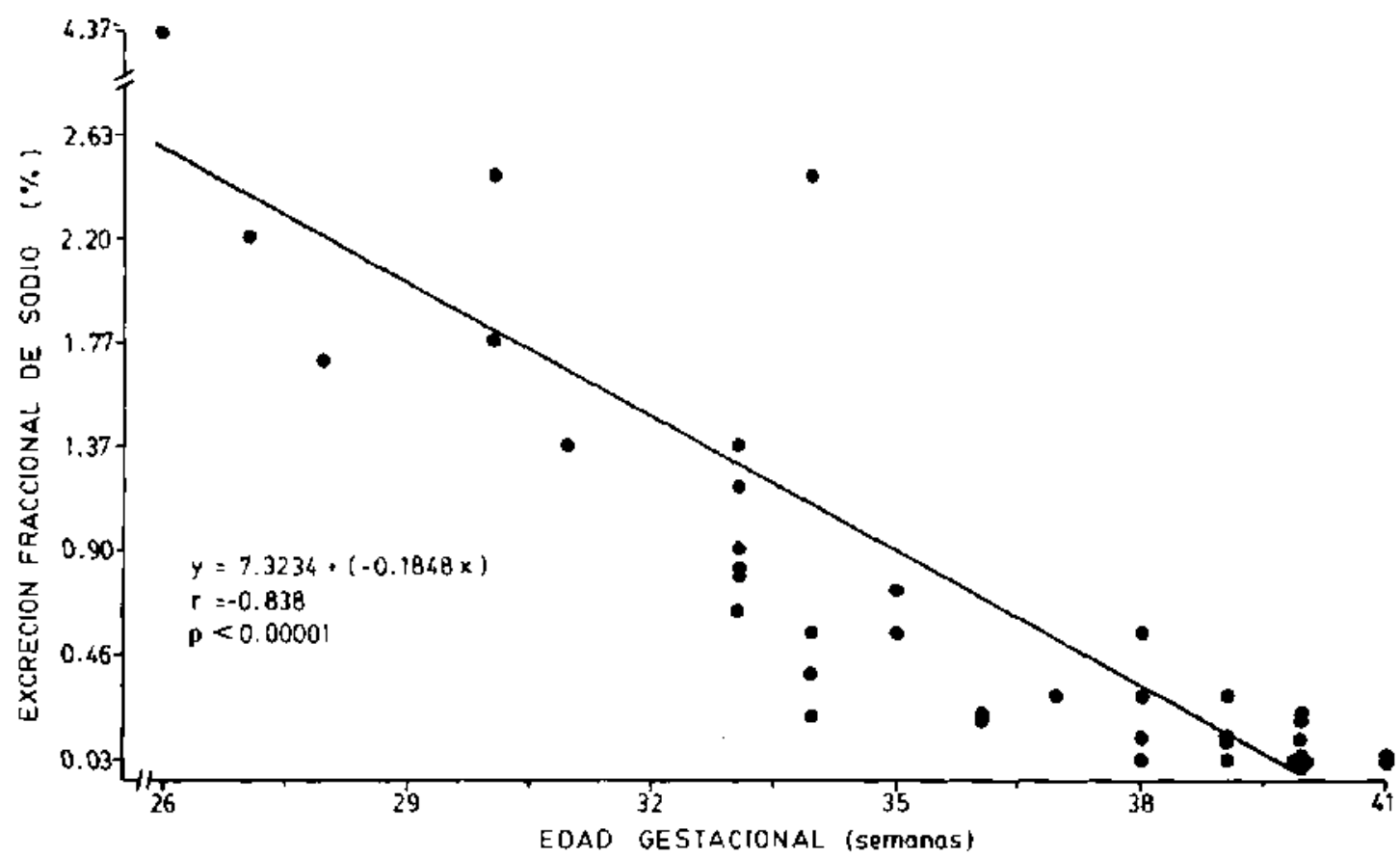

Figura 2: Corroleción de Edad Gestacional y Excteción. Ftaccional de Sodio en 42 RN entre las 24-48 horas de vida. 


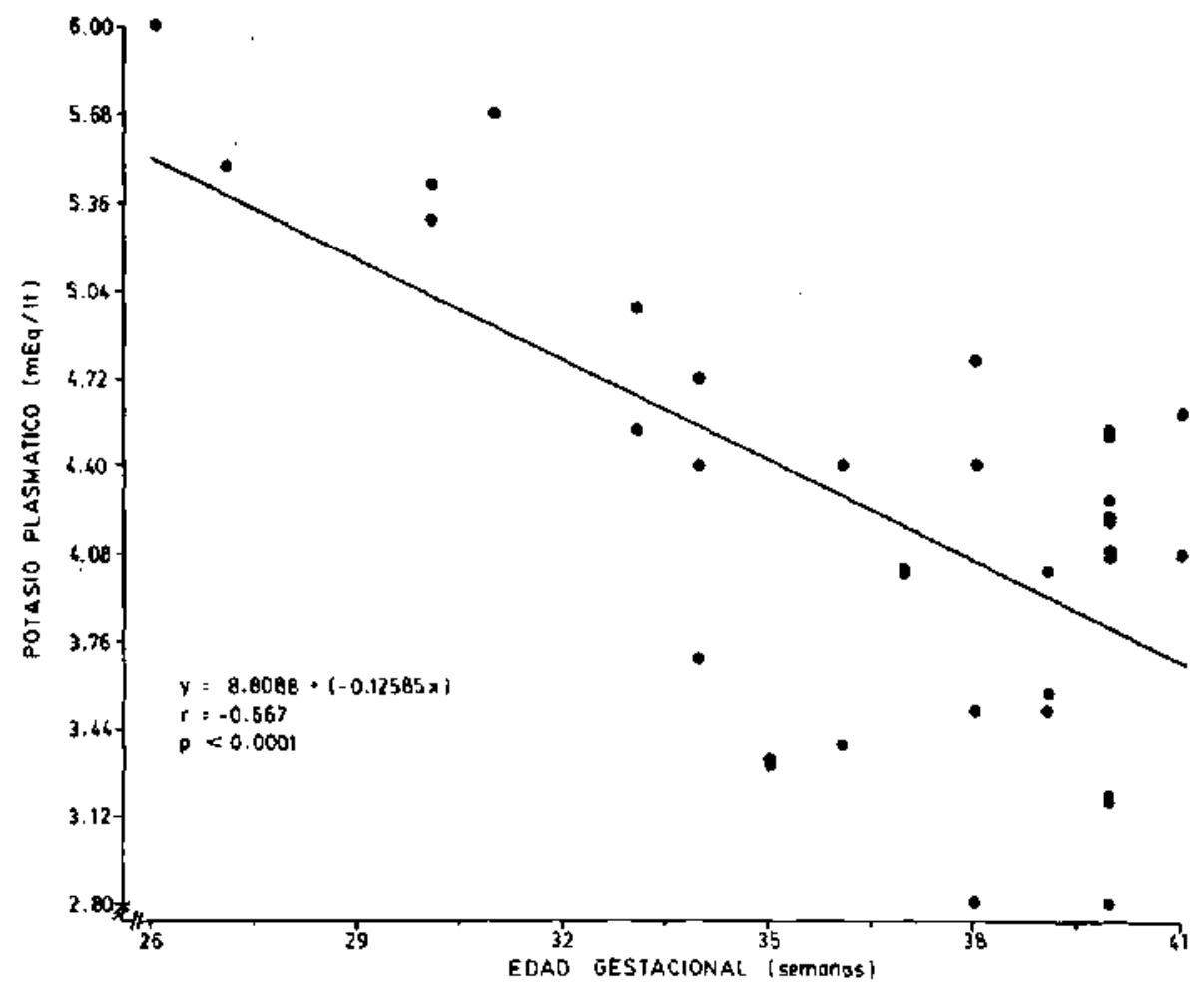

Figura 3: Correlación de Edad Gestacional y Nivel de Potasio Plasmático en 42 RN entre las 24-48 horas de vida.

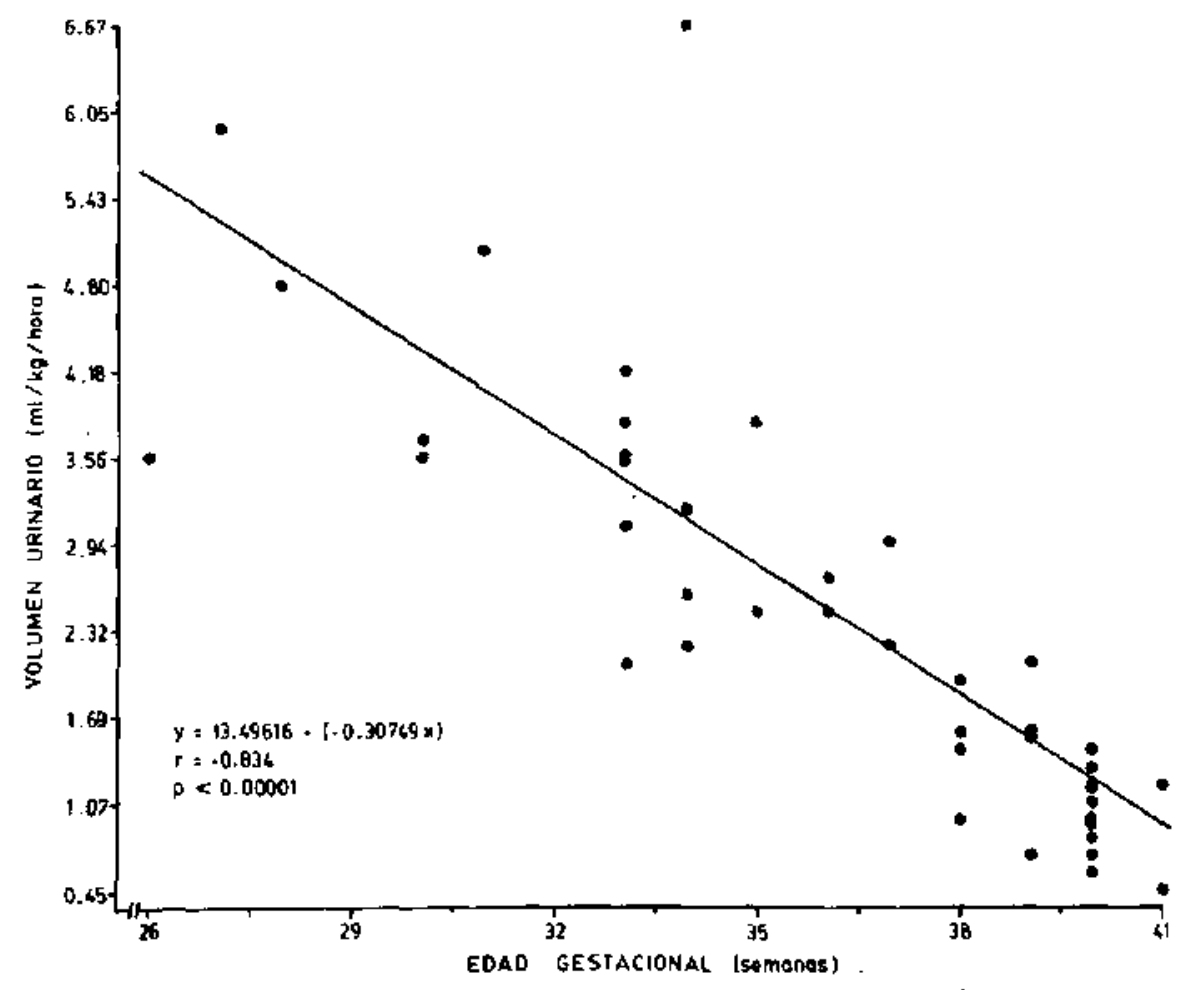

Figura 4: Correlación de Edad Gestacional - Volumen Urinario ( $\mathrm{ml} / \mathrm{kg} /$ hora) entre las $24-48$ horas de vida. 


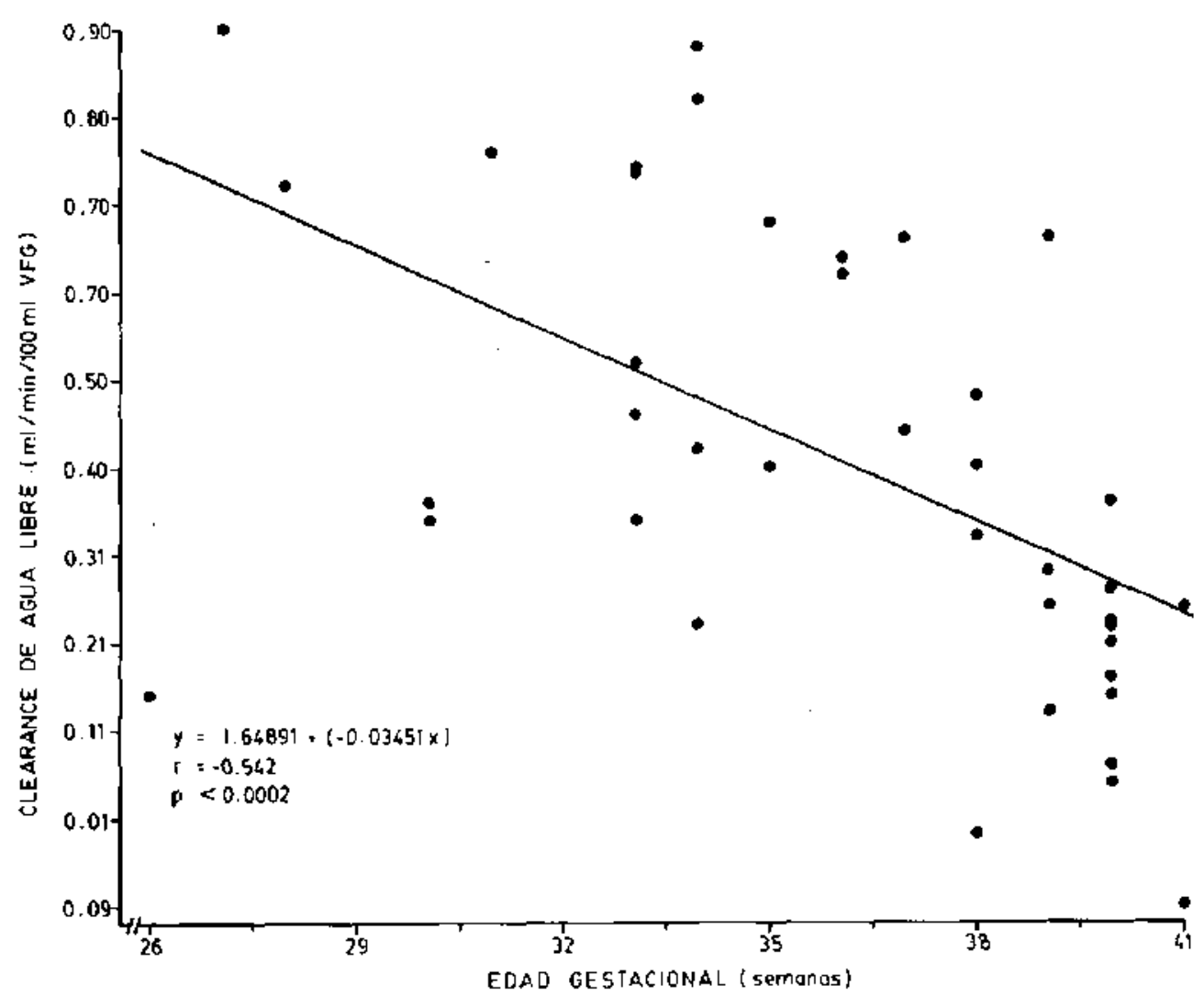

Figura 5: Correlación de Fuad Gestacional y Depuración (clearence) de Agua Libre entre las 24-48 horas de vida.

más bajo en el grupo RNT; observándose una relación inversa con edad gestacional $(\mathrm{r}:-0.834$; p <0.00001) (Figura 4). Así también en el gnupo RNT, la osmolaridad urinaria fue más alta y la depuración de agua libre más baja en comparación al grupo RNPT. Al correlacionar estas dos uj]timas variables con edad gestacional. sólo se alcanzó significación estadística con la depura. ción de agua libre (r: $-0.542 ; \mathrm{p}<0.0002$ ), (Figura 5).

\section{DISCUSION}

Desde los estudios preliminares de Barnett 26 se sabe que la velocidad de filtración giomerular en el recién nacido es menor que en el adulto, aún cuando se efectúe la corrección correspondiente por área de superficie corporal7.

Estudios posteriores han estimado la velocidad de filtración glomerular a través de la depuración de inulina 2,27 y de creatinina ${ }^{1}, 4,5,13$. El alto grado de concordancia en los valores obtenidos con ambas técnicas, las ventajas prácticas de la creatinina y sus escasas limitaciones, han determinado un uso más difundido de esta última ${ }^{5,6}$

Los valores de la depuración de creatinina de este estudio, entre las 24 y 48 horas de vida, son comparables con los establecidos en edades similares en RNT y RNPT por otros autores 1, 2, 2, 4, 5 . Se confirmó también la relación directa del parámetro con la edad gestacional hasta las 34 semanas de gestación, correspondiendo al momento en que se completa la nefrogénesis ${ }^{2,3,4}$. Las diferencias encontradas desde las 35 semanas hasta el término de la gestación reflejan más bien un incremento paralelo de la función renal en relacjón con aumento de la masa renal ${ }^{3}$, como queda demostrado por la correlación existente entre la velocidad de filtración glomerular y peso corporal en este tramo de edad gestacional.

En el RNT la velocidad de filtración glomenjlar aumenta significativamente, alcanzando ya a las 2 semanas el doble del valor de los primeros días de vida $2,5,9,10$. En el RNPT esta respuesta es de menor magnitud ${ }^{5}$, reflejando que los glomérulos más inmaduros responden en menor 
grado a los cambios hemodinámicos que determina el nacimiento.

Es probable que los valores obtenidos en este estudio para los dos grupos de recién nacidos reflejen más bien diferencia de maduración renal fetal, sin desconocer que algunos de los cambios impuestos por la vida extrauterina, podrian estar ya influenciando estos resultados.

La capacidad de reabsorción tubular de sodio, fue proporcional a la edad gestacional en este estudio, confirmando experiencias similares ${ }^{4,28}$. En el grupo RNT, la excreción fraccional de sodio estuvo muy por debajo del $1 \%$, lo que representa un mecanismo adaptativo a la condición de crecimiento tisular, mediado a través de niveles séricos elevados de renina y aldosterona circulante $14,15,16$.

El escape urinario de sodio en el RNPT, especialmente bajo las 34 semanas de gestación, parece obedecer a un doble mecanismo. Por un lado, una menor capaciodad de reabsorción tubu. lar. prokimal de sodio ${ }^{13,16}$; y por otro, una relativa refractariedad del túbulo distal a la acción de aldosterona ${ }^{15}, 17$, De allí la tendencia del RNPT muy inmaduro, a caer en balance negativo de sodio de no mediar una adecuada suplementación de este catión.

No hemos encontrado referencias respecto al comportamiento del potasio plasmático en relación con edad gestacional. En este estudio se demostró una relación inversa muy significativa, que pudiera explicarse por una menor capacidad de secreción de potasio en el túbulo más inmaduro, sin embargo, no hubo diferencias significatjvas en excreción urinatia de potasio entre ambos grupos de recién nacidos, que apoye esta hipótesis.

La orina fetal es hipotónica respecto al plasmall y la capacidad del recièn nacido para concentrar la orina es limitada respecto a edades posteriores 9,12 . Esta limitación, más evidente a menor edad gestacional, se relaciona con el proceso de maduración de los mecanismos de concentración renal; específicamente con un bajo gradiente osmolar' corticomedular determinado por menor capacidad de transporte tubular en el asa ascendente de Henle ${ }^{21}$ y menor reciclaje de urea a nivel medular ${ }^{12}$, a lo que se suma un flujo sanguineo medular aumentado 22 . Por otra parte, la inmadurez del sistema adenil ciclasa tubular es responsable de menor formación de AMP cíclico tubular en respuesta a vasopresina $18,19$.

En el presente estudio, las diferencias en osmolaridad urinaria entre ambos grupos y la correlación inversa observada entre edad gestacional con volumen urinario y depuración de agua libre respectivamente, corroboran lo anteriormente expuesto $y$ explican parcialmente la mayor necesidad de aporte hídrico mientras menor es la edad gestacional del recién nacido.

\section{RESUMEN}

Se estudiaron 42 recién nacidos normales de ambos sexos y diferentes edades gestacionales (rango: 26 a 41 semanas) entre las 24 y 48 horas de vida con el propósito de evaluar la velocidad de filtración glomerular y la excreción renal de agua y electrolitos. La depuración de creatinina se relacionó directamente con edad gestacional (r: 0.67). La excreción fraccional de sodio se relacionó inversamente con la edad gestacional $(r:-0.84)$. No hubo correlación entre excreción urinaria de potasio y edad gestacional, pero se encontró correlación inversa entre esta última y los niveles de potasio plasmático ( $\mathrm{r}:-0.67)$. El volumen urinario y la excreción de agua libre presentaron una correlación inversa con la edad gestacional ( $\mathrm{r}:-0.83$ y r: -0.54 ). Las diferencias encontradas entre el grupo de recién nacidos de término y pretérmino para estos parámetros, reflejan fundamentalmente diferencias en el gradiente madurativo funcional renal, aunque a las 24 horas de vida factores hemodinámicos postnatales pudieran influenciar estos resultados.

\section{AGRADECIMIENTOS}

Los autores desean expresar su reconocimiento a los doctores Carlos Vio, Sergio Mezzano e Italo Caorsi por la revisión crítica del presente trabajo.

\section{REFERENCIAS}

1. Sertel, $H_{\text {; }}$ Scopes, J.: Rates of creatinine clearance in babies less than one week of age. Arch. Dis. Chil. 48: $717,1973$.

2. Guignard, J.P.; Torrado, A.; Da Cunha, O.; et al.: Glomerular filtration rate in the first three weeks of life, J. Pediatu. 87: 268, 1975.

3. Guignard, J.P.: Renal function in the newborn infant. Pediatr. Clin. North Amr. 29: 777, 1982.

4. Sieget, $S . R_{+} ; O h, W$ : Renal function as a marker of human fetal maturation. Acta Paediatr. Scand. 65: $481,1976$.

5. Aperia, A.; Broberger, $O$,; Elinder, G.; et al.: Postratal changes in glomerular filtration rate in preterm and full torm infants. En: The Kidney during Development. Spitzer, A. (ed.). Masson Publishing U.S.A. Inc. N.Y, 1982.

6. Stonestreet, B.S.; Bell, E.F. Oh, W: Validity of endogenous creatinine clearance in low birth weight infants. Pediatr. Res. 13: 1012, 1979. 
7. West. J.R.; Smith, H.W.; Chasis, H.: Glomerular filtration rate, efective renal blood flow, and maximal tubular excretory capacity in infancy. $J$. Pediatr. 32: 10, 1948.

8. Ross, B., Cowett, R.M.; $O h, W .:$ Renal function of low birth weight infants during the first wo months of life. Pediatr. Res 11: 1162, 1977.

9. Spitzer, A.: Renal physiology and functional development. Er: Pediatric Kidney Disease. Edelmann CH.M. (Ed.) Littie, Brown and Company, Boston, 1978 .

10. Spitzer, A.: Factor underlying the increase in glomerular filtration ouring postnatal development. En: The Kidney during Development. Spitzer, A.; (ed.). Masson Publishing U.S.A., Inc. N.Y., 1982.

11. Smith, F.G.; Adems, F.H.; et al.: Studies of renal function in the intact fetal lamb. Am. J. Obstet. Gy necol. 96: 240, 1966.

12. Edelmann, CH.M. Jr.; Bornett, H.L.: Role of the kidney in water metabolism in young infants. Physiologic and clinical considerations. J. Pediatr. S6: 154,1960 .

13. Rodriguez-Soriano, $J_{\text {.; }}$ Vallo, A.; Oliveros, $\boldsymbol{R}_{\text {; }}$ et oL: Renal handling of soditum in pjemature and full term neonates: A study using clearance methods during water diuresis. Pediatr. Res. 17: $1013,1983$.

14. Dillon, M.J.; Gillin, M.E.A.; et al.: Plasma renin activity and aldosterone concentration in the human newborn, Arch. Dis. Child. 51: 537, 1976.

15. Sulyok, E.; Nemeth, M.; Tenyi, I.; et al: Postnatal development of Renin-Angiotensin Aldosterone System (RAAS), in Ielation to ejectrolyte balance in premature infants. Pediatr. Res. 13: 817, 1979.

16. Sulyok, E.; Varga, F.; Jobst, $K$; et al.: On the mechanism of renal sodium handling in newborn infants. Biol. Neona1e 37: 75, 1980.

17. Spitzer, A.: The role of the kidney in sodium homeostasis during maturation. Kidney Int. 21: 539,1982 .

18. Schiondorff, D.; Trizna, W.; Fine, L.G.: Vasopressin activation of adenylate cyclase in isolated collecting tubules of newborn rabbit. En: The Kidney during development, Spitzer, A. (ed.) Masson Publishing U.S.A. Inc. N.Y., 1982.

19. Schlondarf, D.; Weber, H.; et al.: Vasopressin responsiveness of renal adenylate cyclase in newborn rats and rabbits. Am. J. Physiol, 234: F16, 1978.

20. Joppich, R.; Scherer, B.; Webar, P.C.: Renal prostaglandins: Relationship to the development of blowd pressure and concentrating capacity in pretern and fullterm healthy infants. Eur. J. Pediatr. 132: $253,1979$.

21. Zink, H.: Hoster, M.: Maturation of diuting capaci ty in loop of Henle of rat superficial nephrons. Am. J. Physiol. 233: F519, 1977.

22. Edwords, B.R.; Méndez, D.B.; LaRochelle, F.T.: et al.: Postnatal development of urinary concentrating ability in rats: Changes in renal anatomy and neurohypophysial hormones. En: The Kidney during development, Spitzer, A. (ed.). Masson Publishing U.S.A. Inc. N.Y., 1982.

23. Dubowitz, L.M, Dubowitz, V.; Goldberg, C.: Clinical assessment of gestational age in the newborn intant. J. Pediatr. 77: 1, 1970.

24. Domenech i Massons, J.: Bioestadistica. Métodos estadísticos para investigadores. Edit. Herder, Barcelona, 1977.

25. Battaglio, F, Lubchenco. L.O.: A practical classification of newborn infant by weight and gestational age. J. Pediatu. 71: 159, 1967.

26. Barnetr, H.L.; Hare, $K$; McNamort, H.: Hare, $R$. : Measurement of glomerular filitation rate in premature infants. J. Clin. Invest. 27: 691, 1948.

27. Leake, R.D.; Trygstad, C.W.: Glomerular filtration rate during the period of adaptntion to extrauterine life. Pedint. Res. 11: 959, 1977.

28. Aperia, A.; Brobenger, O.: Thoctentus, et al: Development of renal control of salt and fluid homeostasis during the first year of life. Acta Paediatr. Scand. 64: 393, 1975. 